\title{
Combined genetic and imaging diagnosis for two large Chinese families affected with Pelizaeus-Merzbacher disease
}

\author{
Y. Lv ${ }^{1 *}$, L.H. Cao ${ }^{2 *}$, H. Pang ${ }^{3}$, L.N. Lu' ${ }^{2}$, J.L. Li ${ }^{1}$, Y. Fu ${ }^{1}$, S.L. Qi' ${ }^{4}$, Y. Luo ${ }^{2}$ \\ and J. Li-Ling ${ }^{1,4,5}$ \\ 'Department of Medical Genetics, School of Basic Medicine, \\ China Medical University, Shenyang, China \\ ${ }^{2}$ Research Center for Medical Genomics, School of Basic Medicine, \\ China Medial University, Shenyang, China \\ ${ }^{3}$ Shenyang Women and Children's Hospital, Shenyang, China \\ ${ }^{4}$ Sino-Dutch Biomedical and Information Engineering School, \\ Northeastern University, Shenyang, China \\ ${ }^{5}$ Key Laboratory for Bio-Resources and Eco-Environment, Ministry of Education, \\ Institute of Medical Genetics, School of Life Science, \\ Sichuan University, Chengdu, China \\ *These authors contributed equally to this study. \\ Corresponding authors: J. Li-Ling / Y. Luo \\ E-mails: jliling@scu.edu.cn / luoyangcmu@yahoo.com.cn \\ Genet. Mol. Res. 11 (3): 2035-2044 (2012) \\ Received October 17, 2011 \\ Accepted March 5, 2012 \\ Published August 6, 2012 \\ DOI http://dx.doi.org/10.4238/2012.August.6.7
}

ABSTRACT. Pelizaeus-Merzbacher disease (PMD) is a rare $\mathrm{X}$-linked recessive disorder characterized by nystagmus, impaired motor development, ataxia, and progressive spasticity. Genetically defective or altered levels of proteolipid protein (PLP1) or gapjunction alpha protein 12 gene have been found to be a common cause. Here we report on two large Han Chinese families affected with this disease. The probands of both families had produced sons featuring cerebral palsy that had never been correctly diagnosed. 
PMD was suspected after careful analysis of family history and clinical features. Three rounds of molecular testing, including RTPCR, genetics linkage and SRY sequence analyses, in combination with fetal ultrasound and magnetic resonance imaging, confirmed the diagnosis. In Family 1, in addition to two patients, three carriers were identified, including one who was not yet married. Genetic testing indicated that a fetus did not have the disease. A healthy girl was born later. In Family 2, two patients and two carriers were identified, while a fetus was genetically normal. A healthy girl was born later. We concluded that by combining genetic testing and imaging, awareness of the symptoms of PMD and understanding of its molecular biology, there is great benefit for families that are at risk for producing offspring affected with this severe disease.

Key words: Genetic testing; Magnetic resonance imaging; Pelizaeus-Merzbacher disease; PLP1 gene; Prenatal diagnosis

\section{INTRODUCTION}

Cerebral palsy is a relatively common disorder that brings severe burden to affected families because of its serious clinical sequelae. The disease is causatively heterogeneous, with possible causes including birth injury, hypoxia, prenatal infection, metabolic defects, and genetic mutations, which may result in similar symptoms. Clinical counseling for such condition is therefore rather difficult. We hereby report on two large Chinese families with multiple affected members, for whom combined genetic and imaging analysis facilitated successful prenatal diagnosis.

For Family 1, a 31-year-old gravida, together with her mother and first son, came to Genetic Clinics of Shengjing Hospital Affiliated to China Medical University at gestational week $26^{+5}$. The proband complained about having already given birth to two sons, both featuring cerebral palsy. Her first son, now 8 years old, was born at the 38th gestational week after an uncomplicated pregnancy and normal delivery. Roving eye movements were noticed 10 days after birth. The child was later hospitalized for seizures, horizontal nystagmus and hypotonia. On physical examination, he was found to have developmental delay, profound mental retardation, funnel chest, amyotrophy in the lower limbs, and inability to stand without support. $\mathrm{He}$ was predominantly non-verbal but could occasionally speak a few words.

As told, the second son of the proband was affected with similar symptoms, but which were more severe. Nystagmus and seizures were present a few months after birth. At 5 years old, he was still unable to sit without support due to severe hypotonia. He had poor head and trunk control, in addition to severe mental retardation (Table 1). The proband and her husband (aged 33) were screened for chromosomal abnormality at a local hospital, and the results were both normal.

When taking the family history, the proband could only tell that, besides her two sons, she had an elder sister (III) who had died of leukemia at the age of 17. The grandmother ( $\mathrm{II}_{4}$ ), however, added that she herself had produced 6 daughters and one son $\left(\mathrm{III}_{8}\right)$. The son had perished at 3 years old due to symptoms similar to those of $\mathrm{IV}_{1}$ and $\mathrm{IV}_{2}$. Notably, she also had two brothers ( $\mathrm{II}_{1}$ and $\mathrm{II}_{5}$ ) who died at, respectively, around 1 and 30 years old. The two also seemed to have had similar symptoms (Figure 1). 


\begin{tabular}{|c|c|c|c|}
\hline & \multicolumn{2}{|c|}{ Family 1} & \multirow{2}{*}{$\frac{\text { Family } 2}{\mathrm{IV}_{1}}$} \\
\hline & $\mathrm{IV}_{1}$ & $\mathrm{IV}_{2}$ & \\
\hline Age at first symptom & 10 days & Few months & 10 years old \\
\hline Nystagmus & + & + & + \\
\hline Seizures & + & + & - \\
\hline Head control & Normal & Poor & Poor \\
\hline Muscular hypotonia & + & + & + \\
\hline Sit & Poor & Poor & Poor \\
\hline Stand & Poor & Unable & Poor \\
\hline Speak & Poor & Unable & Normal \\
\hline Intelligence & Profound mental retardation & Profound mental retardation & Profound mental retardation \\
\hline
\end{tabular}

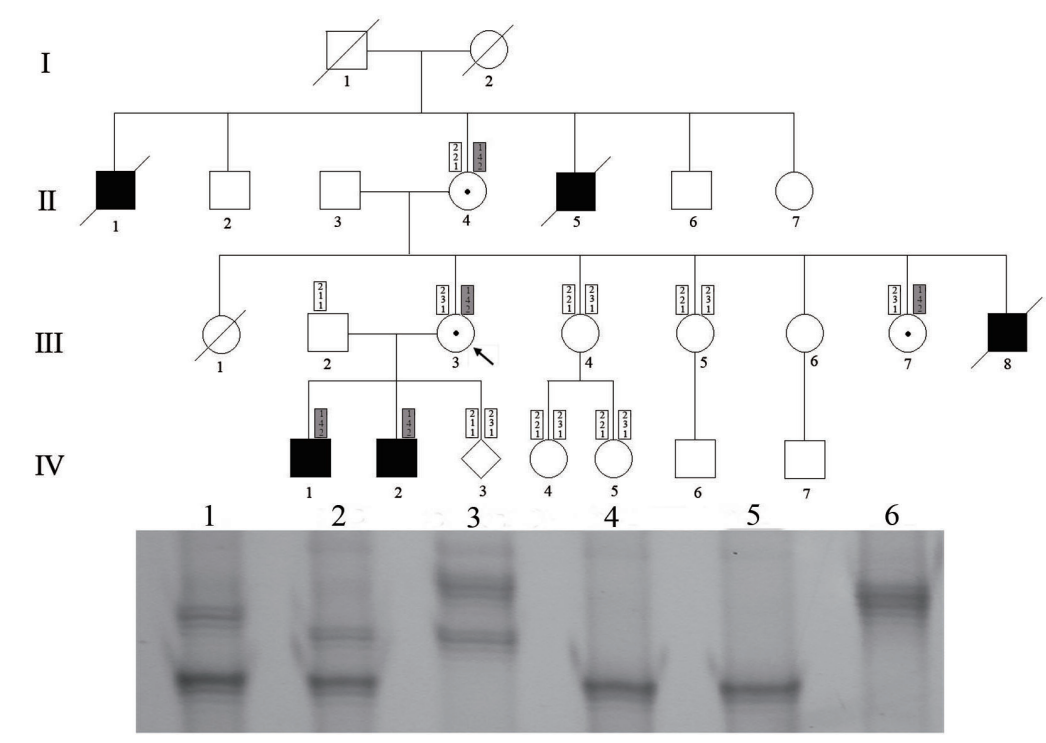

Figure 1. Pedigree and haplotype construction for Family 1. Filled symbols represent affected members, open symbols represent unaffected members. Circles and squares represent females and males, respectively. Dotcentered circles represent female carriers. The arrow identifies the proband. Boxes show the haplotypes of the family members constructed with three polymorphic genetic markers. As shown, haplotype 1-4-2 has probably cosegregated with the disease in the family. The electrophoresis gel at the bottom shows the result of linkage analysis using one of the genetic markers, which mapped within the $P L P 1$ gene. Lane $1=\mathrm{II}_{4}$; lane $2=\mathrm{III}_{3}$; lane $3=\mathrm{IV}_{3}$ (cord blood); lane $4=\mathrm{IV}_{1}$; lane $5=\mathrm{IV}_{2}$; lane $6=\mathrm{III}_{2}$.

Family 2 seemed to have the same disease (Figure 2). The proband, a 40-year-old gravida, had given birth to a son by Caesarean section in 2000. Cerebral palsy and nystagmus were noted in the son. Head magnetic resonance imaging (MRI) indicated that his frontal lobes were poorly formed. On physical examination, the boy showed over-stretched head and was unable to stand without support. He was able to speak, with mental retardation equivalent to $3 \sim 4$ years old.

The proband also had a brother (aged 25) with similar symptoms. He also had an overstretched head. Unstable gait was noticed shortly after birth. MRI revealed cerebral atrophy. He still could not walk after a spinal surgery and was delayed in motor development. The proband also had two uncles $\left(\mathrm{II}_{1}\right.$ and $\left.\mathrm{II}_{3}\right)$ with similar syndromes who had died shortly after birth. 
Although no diagnosis could be made on site, considering the severe burden to these families, peripheral blood samples were taken from each patient as well as non-affected family members. Pedigree analysis suggested that both families had been affected with a single gene disorder featuring X-linked recessive inheritance. Further review of the symptoms had led to the suspicion of Pelizaeus-Merzbacher disease (PMD; MIM 312080), a genetic condition featuring X-linked recessive inheritance. Considering the high risk of recurrence and late stage of pregnancy, prompt action was taken for genetic testing and prenatal diagnosis.
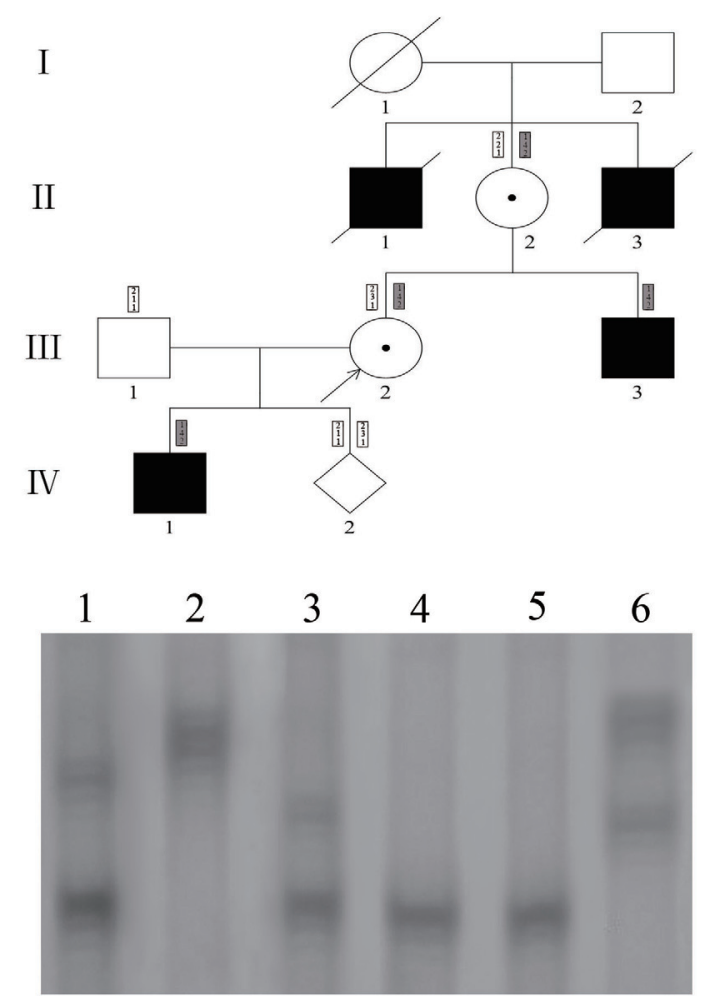

Figure 2. Pedigree and haplotype construction for Family 2. Filled symbols represent affected members, open symbols represent unaffected members. Circles and squares indicate females and males, respectively. Dot-centered circles represent female carriers. The arrow identifies the proband. Boxes show the haplotypes of the family members constructed with three polymorphic genetic markers. The electrophoresis gel at the bottom shows the result of linkage analysis using one of the genetic markers, which mapped within the PLP1 gene. Lane $1=\mathrm{II}_{2}$; lane $2=\mathrm{III}_{1}$; lane $3=\mathrm{III}_{2}$; lane $4=\mathrm{III}_{3} ;$ lane $5=\mathrm{IV}_{1}$; lane $6=\mathrm{IV}_{2}$ (amniotic fluid).

\section{SUBJECTS AND METHODS}

\section{Sample collection}

With informed consent obtained, peripheral venous blood was sampled from both families, which was anti-coagulated with sodium citrate. When results from the first round of 
testing became available, umbilical cord blood sampling of the proband from Family 1 was ordered and performed at $30^{+2}$ gestational week. For Family 2, an amniotic fluid sample was obtained at 30th gestational week. Peripheral venous blood was sampled from members of both families when available. All testing was granted by the Ethics Committee of Shengjing Hospital affiliated with China Medical University.

\section{Extraction of genomic DNA}

Genomic DNA was extracted from peripheral blood, cord blood and amniotic fluid samples with either a standard proteinase $\mathrm{K}$ digestion and phenol-chloroform extraction procedure or a Blood Genomic Prep Minispin kit (GE, USA).

\section{Real-time quantitative PCR}

To detect potential duplications or deletions of the $P L P 1$ gene, 3 pairs of primers (for exons 1, 4 and 7, respectively, Table 2) were designed according to its sequence (GenBank accession No. NC_000023.9) and synthesized (GenScript Corporation, China). The testing was performed on a real-time quantitative PCR instrument (ABI, USA). In practice, the reaction was carried out using a TaKaRa SYBR ${ }^{\circledR}$ Premix Ex Taq ${ }^{\mathrm{TM}}$ kit (TaKaRa, Japan). The $20-\mu \mathrm{L}$ reaction system comprised $400 \mathrm{nM}$ primers, $50 \mathrm{ng}$ genomic DNA and $10 \mu \mathrm{L}$ SYBR Premix Ex $\mathrm{Taq}^{\mathrm{TM}}$. The cycling was designed as $95^{\circ} \mathrm{C}$ for $30 \mathrm{~s}, 95^{\circ} \mathrm{C}$ for $5 \mathrm{~s}$, and $60^{\circ} \mathrm{C}$ for $34 \mathrm{~s}$ for 40 cycles. Each sample was measured in 3 separate tubes, and each experiment was run in triplicate.

\begin{tabular}{|c|c|}
\hline Primer & Sequence \\
\hline \multicolumn{2}{|c|}{ For real-time PCR } \\
\hline qPLP1-1F & 5'-ACAAAGTCAGCCACAAAGCAGA-3' \\
\hline qPLP1-1R & 5'-CGAACCCCAAAGTTGGAAATT-3' \\
\hline qPLP1-4F & 5'-GTACATTTACTTCAACACCTGGACC-3' \\
\hline qPLP1-4R & 5'-CCAAAGCACCCGTACCCTAA-3' \\
\hline qPLP1-7F & 5'-AAGGGATTTGAGGAGGGAGTG-3' \\
\hline qPLP1-7R & 5'-TAAGGACGGCAAAGTTGTAAGTG-3' \\
\hline $\mathrm{B}$-actin $\mathrm{F}$ & 5'-ACTGTGCCCATCTACGAGG-3' \\
\hline B-actin R & 5'-ATGTCACGCACGATTTCCCG-3' \\
\hline \multicolumn{2}{|c|}{ For linkage analysis } \\
\hline mPLP1F & 5'-ATTGGGAAGGTCATCTCAG-3' \\
\hline mPLP1R & 5'-TCATGTGAGCCAGTTCTTG-3' \\
\hline mPLP2F & 5'-GCTAGGACTAGAAGTGCGTACC-3' \\
\hline mPLP2R & 5'-GGCAGTGACAAATGTTACTAGAGC-3' \\
\hline mPLP3F & 5'-CCTGCCAAGTTCTCAGCTCTG-3' \\
\hline mPLP3R & 5'-CCAACAGTCTGCATCTTACTATTAG-3' \\
\hline mPLP4F & 5'-GGAGACTGACCTGAGGAACAAG-3' \\
\hline mPLP4R & 5'-CACGGCAACAGCATCTGGAC-3' \\
\hline
\end{tabular}

Results of real-time PCR were processed using a $\Delta \Delta C t$ method, where $C t$ refers the number of cycles for the product to reach the threshold where fluorescence becomes detectable. The method features relative quantification, which is calculated as $\Delta \Delta C t=\left(C t_{\text {target gene }}\right.$ - $\left.C t_{\text {internal control }}\right)_{\text {experimental group }}-\left(C t_{\text {target gene }}-C t_{\text {internal control }}\right)_{\text {control group }}$. Relative copy number of (experimental group/control group) $=2^{-\Delta \Delta C t}$. Using a normal male DNA as the standard, the expected ratio was 1 for a single PLP1 gene copy (normal males), 2 for two PLP1 gene copies (males 
carrying the duplication or normal females) and 3 for three PLPI gene copies (female carriers). The copy number was considered to have increased when $2^{-\Delta \Delta C t}>1.75$. Calculations, statistical analyses and construction of column diagrams were carried out using functions of the Excel software (Microsoft Corporation, USA).

\section{SRY sequence analysis}

To determine the gender of the fetus, PCR specific for the SRY sequence (292 bp) was performed with male and female subjects from the family as controls. The sequences of the primers were: forward: 5'-GGAAGGCGAAGATGCTG-3', and reverse: 5'-GAAAGTGAGGGCTGTAAG-3' .

\section{STR linkage analysis}

Genomic DNA was extracted from the blood samples of the proband, her husband, two affected sons and the fetus of Family 1, as well as the proband, her husband, the affected son and the fetus of Family 2. Four simple tandem repeat (STR) markers within or flanking the PLP1 gene (centromere-DXS8096, CA-PLP (within the first intron of PLP1), DXS1191, DXS8075-telomere) were amplified under standard conditions. The PCR products were separated by electrophoresis on a $8 \%$ denaturing polyacrylamide gel, and allele fragments were detected with routine silver staining. On the basis of the individual's genotype and kinship, the haplotypes were deduced according to the law of Mendelian inheritance. Transmission of the mutation-carrying $\mathrm{X}$ chromosome in the two families was traced through linkage analysis.

\section{Imaging analysis}

Gender and potential malformations of the fetus from Family 1 were again checked with ultrasound. Fetal magnetic resonance imaging was performed on a Neusoft Superstar 0.35T MRI System.

\section{RESULTS}

\section{Family 1}

As indicated by RT-PCR, the proband, her mother and her first son $\left(\mathrm{III}_{3}, \mathrm{II}_{4}\right.$ and $\mathrm{IV}_{1}$ ) carried, respectively, 3,3 and 2 copies of the PLP1 gene. This confirmed that, while the first two subjects were carriers, the first son had PMD. Blood samples from the eldest younger sister of the proband and her daughters ( $\mathrm{III}_{4}, \mathrm{IV}_{4}$ and $\left.\mathrm{IV}_{5}\right)$, together with that of her youngest sister $\left(\mathrm{III}_{7}\right)$, were analyzed in blind fashion. As revealed, while the first three subjects were all negative for the duplication, the youngest sister was in fact a carrier. The fetus $\left(\mathrm{IV}_{3}\right)$ carried 2 copies (Figure 3A). On the other hand, no PCR fragment specific for the SRY sequence was derived from the fetus, suggesting it to be a female (Figure 4). Linkage analysis also indicated that the fetus did not carry the same $\mathrm{X}$ chromosome as its two brothers (Figure 1). Combined results for the above indicated that the fetus was a healthy female and not a carrier. 

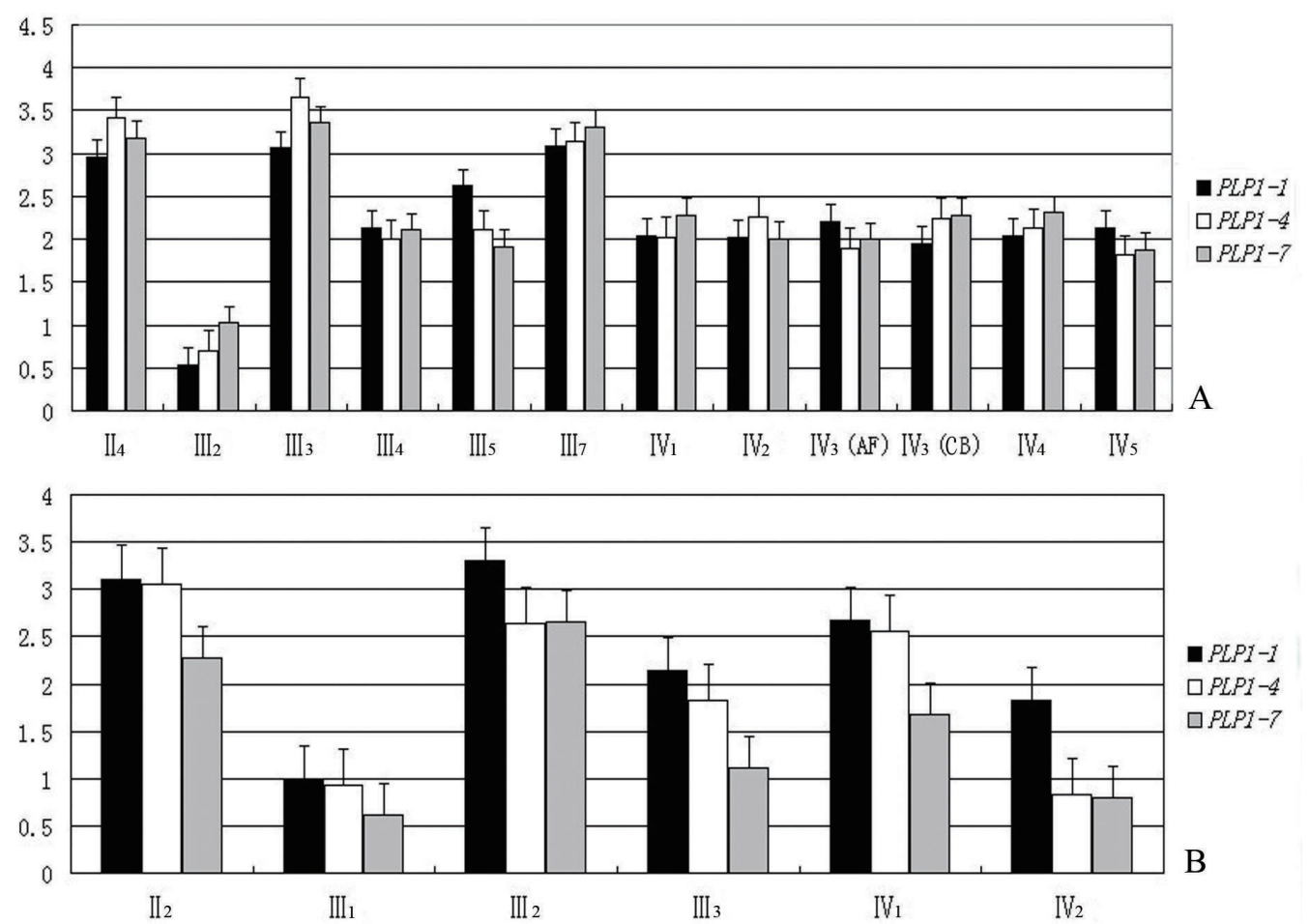

Figure 3. Results of real-time PCR. Exons 1, 4 and 7 of the PLP1 gene were respectively assayed with real-time PCR. Relative copy numbers are represented by the height of columns. A. RT-PCR results for $\mathrm{II}_{4}, \mathrm{III}_{2}, \mathrm{III}_{3}, \mathrm{III}_{4}, \mathrm{III}_{5}$, $\mathrm{III}_{7}, \mathrm{IV}_{1}, \mathrm{IV}_{2}, \mathrm{IV}_{3}$ (AF, amniotic fluid), $\mathrm{IV}_{3}\left(\mathrm{CB}\right.$, cord blood), $\mathrm{IV}_{4}$ and $\mathrm{IV}_{5}$ of Family 1. B. RT-PCR results for II $\mathrm{I}_{2}$, $\mathrm{III}_{1}, \mathrm{III}_{2}, \mathrm{III}_{3}, \mathrm{IV}_{1}$ and $\mathrm{IV}_{2}$ (amniotic fluid) of Family 2.

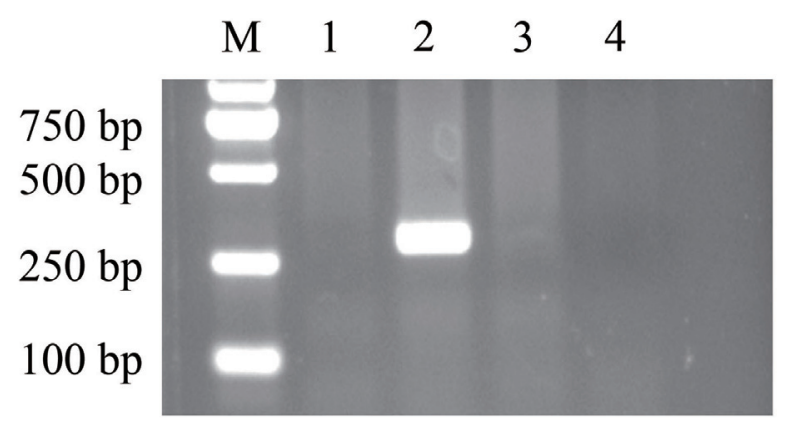

Figure 4. Results of SRY sequence analysis. Lane $M=$ DNA marker; lane $1=$ female; lane $2=$ male; lane $3=\mathrm{IV}_{3}$ of Family 1; lane $4=\mathrm{IV}_{2}$ of Family 2 . Both fetuses were found to be females.

At 37th gestational week, the proband brought both sons back for an MRI scan. With written informed consent obtained, fetal MRI imaging was carried out (Figure 5). Furthermore, with permission obtained from the Ethics Committee of the hospital, the gender of the 
fetus was again checked with ultrasound, which confirmed the fetus to be a female. MRI scan of the first son showed severe leukodystrophy in the brain (Figure 5). For the second son ( $\left.\operatorname{IV}_{2}\right)$, due to inadequate sedation with chloral hydrate, this procedure was abandoned.

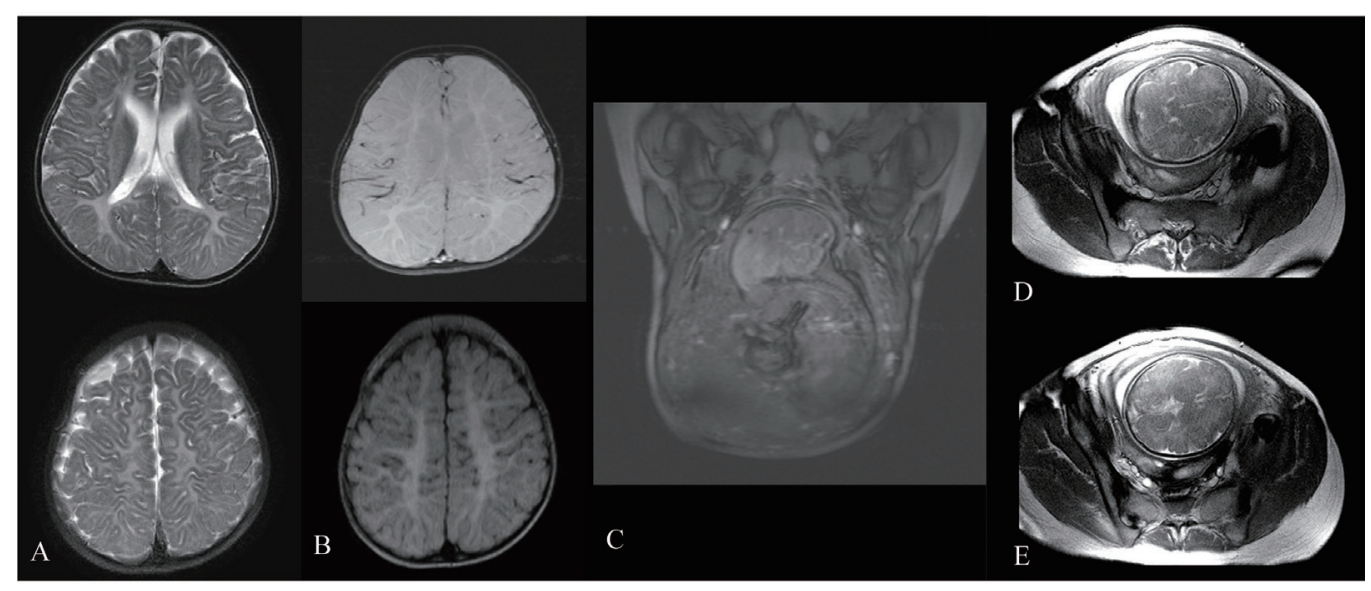

Figure 5. MR images of $\mathrm{IV}_{1}$ and $\mathrm{IV}_{3}$ of Family 1. For $\mathrm{IV}_{1}$, as shown in $\mathbf{A}$ and $\mathbf{B}, \mathrm{T} 2$ signal from the paraventricular white matter appeared increased, suggesting edema. Low T1 and high T2 signals surrounding the pons suggested developmental anomaly. No myelination was seen in the white matter. The overall results seemed to suggest hereditary metabolic disease of the brain. Magnetic resonance imaging: C. FFE-25/7.0; D. B-FFE-9.0/4.5; E. one backup of the fetus $\left(\mathrm{IV}_{3}\right)$ of Family 1 at gestation week 37 . No salient structural anomalies were seen, but due to the poor quality of the image, no accurate diagnosis could be made.

At 40th gestational week, the proband gave birth to a healthy female baby who weighed $3.6 \mathrm{~kg}$ and measured $50 \mathrm{~cm}$ in height. As of the date of this report, the girl has been developing well.

\section{Family 2}

As shown in Figure 3, RT-PCR indicated that the proband, her mother and her son $\left(\mathrm{III}_{2}, \mathrm{II}_{2}\right.$ and $\left.\mathrm{IV}_{1}\right)$ carried, respectively, 3,3 and 2 copies of the PLP1 gene. This confirmed that while the first two individuals were carriers, the son had PMD. A further round of testing indicated that the father ( $\left.\mathrm{III}_{1}\right)$ carried 1 copy of the PLP1 gene, while the son and the fetus carried 2 copies (Figure 3B). No SRY sequence was detected in the fetus (Figure 4), suggesting it to be a female. Linkage analysis also confirmed that the fetus did not carry the same $\mathrm{X}$ chromosome as its brother (Figure 2). At 40th gestational week, the proband gave birth to a healthy female baby.

\section{DISCUSSION}

PMD is a form of dysmyelinating leukodystrophy that primarily affects the white matter of the central nervous system (CNS). This disease is characterized by nystagmus, dystonia, impaired motor development, ataxia, progressive spasticity, and mild cognitive delay. 
The prevalence of PMD has been estimated to be around 1/200,000 to $1 / 500,000$ in the USA, though no such data are yet available in China. Clinically, this disease can be classified into three subtypes according to its severity: connatal, transitional, and classical. A milder spastic paraplegia type 2 (SPG2; MIM 312920) has also been identified as an allelic disorder. In some cases, it may be difficult to discriminate between complicated SPG2 and the mild form of PMD.

In 1986, the causative gene for PMD, PLP1, was mapped at Xq22. The gene spans 17 $\mathrm{kb}$ and contains 7 coding exons. By alternative splicing of exon 3 , the gene encodes the major structural component of myelin, proteolipid protein (PLP), in addition with a splicing isoform DM20 which differs by 35 amino acids in the intracytoplasmic loop. Both transcripts express abundantly in oligodendrocytes of the CNS. Pathogenetically, deficiency in PLP synthesis can result in reduction of myelination in the CNS (Willard and Riordan, 1985; Mattei et al., 1986). As confirmed by recent research, duplication, point mutations (missense, nonsense or splicing types) and large deletion of the PLP1 gene respectively account for $60 \sim 70,15 \sim 20$, and 1 2\% of PMD cases (Inoue et al., 2001, 2002). The role of PLP/DM20 proteins has not been fully elucidated, though it seems that they have a function in the stabilization of the myelin sheaths as well as axonal transport. In humans, expression of PLP1 gene predominates in the oligodendrocytes and correlates with the initiation of myelination in the third trimester of pregnancy. PLP/DM20 constitutes up to $\sim 50 \%$ of the myelin proteins. PLP transcript prevalently expresses in adult myelinating oligodendrocytes, while DM20 is the predominant isoform at embryonic stages. In PMD patients, PLP1 gene duplication can cause over-expression and altered balance of PLP/DM20 splicing, and similar results have been obtained in transgenic mice overexpressing PLP1. In vitro experiments and transgenic mouse models have both indicated that over-expression of PLP can lead to the formation of late endosomal/lysosomal accumulations of cholesterol and PLP, which is accompanied by mis-trafficking of raft components necessary for normal myelination (Krämer-Albers et al., 2006).

Genetic mutations so far identified in the $P L P 1$ gene have been highly variable, which include duplications, point mutations and deletions. In $60 \sim 70 \%$ of cases, genomic rearrangements resulting in PLP1 gene duplication have been found, while $10 \sim 25 \%$ of cases are due to point mutations located mostly in protein-encoding regions. Such mutations often cause replacement of amino acids, and can also affect the splicing sites and non-encoding areas. Since affected families often carry different point mutations, copy number determination and mutation detection for the $P L P 1$ gene should provide a comprehensive and effective strategy for identifying potential mutations (Bonnet-Dupeyron et al., 2008).

With regard to genotype-phenotype correlation, duplications of the PLP1 gene in general can result in classical or transitional type of PMD, while point mutations may show a wider clinical spectrum ranging from the most severe connatal form to SPG2, depending on which part of the protein is affected. In contrast, in case of null or complete deletion of the PLP1 gene, the phenotype is relatively mild. Notably, some female carriers may exhibit transient signs in childhood or late-onset PMD. Compared with null alleles or nonsense mutations, PLP1 duplications are as likely to confer symptoms in female carriers as missense mutations, which may be related to specific skewed X-inactivation. In the present study, all female carriers appeared to be normal, though further study may be necessary to reveal subtle mental defects (Inoue, 2005).

As male gender preference still prevails to certain extent in the country, prenatal gen- 
der detection is currently prohibited by law in China. However, considering the high risk for any males born to these families, we have obtained special permission from the Ethics Committee of the hospital for determining the gender of the fetus. Despite its limited resolution, ultrasonography has proven to have great value for detection of fetal anomalies as well as the gender. To ensure accurate detection, SRY sequence detection in combination with repeated ultrasound scans were carried out. STR microsatellite markers were also used for tracing the mutant $\mathrm{X}$ chromosome in the family through linkage analysis.

In the present study, fetal MRI was used as a complementary diagnosis, considering the potential presence of incomplete myelination. However, this has proven to be of limited value since myelination under normal circumstances is only completed by 2 years of age (Edgar et al., 2004). Taken together, our cases have demonstrated that, with awareness of its clinical features and molecular pathology, combined molecular testing and fetal imaging, can bring great benefit to families whom have been suffering from a severe disease such as PMD.

\section{ACKNOWLEDGMENTS}

Research supported by grants from the National Natural Science Foundation of China (\#81072899), the National 863 Program of China (\#2007AA02Z440), the Fundamental Research Funds for the Central Universities (\#N110804002), and the Overseas Teacher Project of Ministry of Education and State Administration of Foreign Experts Affairs (\#MS2011DBDX021). We thank our patients and their families for participating in this study. Particular thanks also go to Neusoft Medical System, Co. Ltd., for providing the MRI scan.

\section{REFERENCES}

Bonnet-Dupeyron MN, Combes P, Santander P, Cailloux F, et al. (2008). PLP1 splicing abnormalities identified in Pelizaeus-Merzbacher disease and SPG2 fibroblasts are associated with different types of mutations. Hum. Mutat. 29: $1028-1036$.

Edgar JM, McLaughlin M, Barrie JA, McCulloch MC, et al. (2004). Age-related axonal and myelin changes in the rumpshaker mutation of the PLP gene. Acta Neuropathol. 107: 331-335.

Inoue K (2005). PLP1-related inherited dysmyelinating disorders: Pelizaeus-Merzbacher disease and spastic paraplegia type 2. Neurogenetics 6: 1-16.

Inoue K, Kanai M, Tanabe Y, Kubota T, et al. (2001). Prenatal interphase FISH diagnosis of PLP1 duplication associated with Pelizaeus-Merzbacher disease. Prenat. Diagn. 21: 1133-1136.

Inoue K, Osaka H, Thurston VC, Clarke JT, et al. (2002). Genomic rearrangements resulting in PLPI deletion occur by nonhomologous end joining and cause different dysmyelinating phenotypes in males and females. Am. J. Hum. Genet. 71: 838-853.

Krämer-Albers EM, Gehrig-Burger K, Thiele C, Trotter J, et al. (2006). Perturbed interactions of mutant proteolipid protein/DM20 with cholesterol and lipid rafts in oligodendroglia: implications for dysmyelination in spastic paraplegia. J. Neurosci. 26: 11743-11752.

Mattei MG, Alliel PM, Dautigny A, Passage E, et al. (1986). The gene encoding for the major brain proteolipid (PLP) maps on the q-22 band of the human X chromosome. Hum. Genet. 72: 352-353.

Willard HF and Riordan JR (1985). Assignment of the gene for myelin proteolipid protein to the X chromosome: implications for X-linked myelin disorders. Science 230: 940-942. 